\title{
Prosthesis-patient mismatch is less frequent and more clinically indolent in patients operated for aortic insufficiency
}

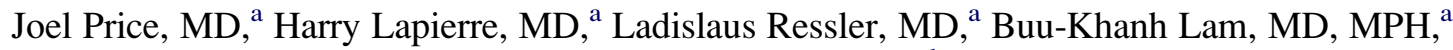 \\ Thierry G. Mesana, MD, PhD, ${ }^{\mathrm{a}}$ and Marc Ruel, MD, $\mathrm{MPH}^{\mathrm{a}, \mathrm{b}}$
}

\begin{abstract}
Objective: To date, no study has focused on the incidence and effects of prosthesis-patient mismatch in patients requiring aortic valve replacement for aortic insufficiency. We hypothesized that the incidence and implications of prosthesis-patient mismatch in patients with aortic insufficiency might be different than for aortic stenosis or mixed disease because the annulus is generally larger in aortic insufficiency and left ventricular remodeling might differ.
\end{abstract}

Methods: Ninety-eight patients with lone aortic insufficiency $(\geq 3+$ with a preoperative mean gradient $<30 \mathrm{~mm}$ $\mathrm{Hg}$ ) were followed over $7.7 \pm 4.3$ years (maximum, 17.5 years) with clinical and echocardiographic assessments. They were compared with 707 patients who had aortic valve replacement for aortic stenosis or mixed disease. Prosthesis-patient mismatch was defined as an in vivo indexed effective orifice area of $0.85 \mathrm{~cm}^{2} / \mathrm{m}^{2}$ or less.

Results: Compared with patients with aortic stenosis/mixed disease, patients with aortic insufficiency had approximately half the incidence of prosthesis-patient mismatch $(P=.003)$. Patients with prosthesis-patient mismatch had significantly higher transprosthesis gradients postoperatively. An independent detrimental effect of prosthesis-patient mismatch on survival was observed in patients with aortic stenosis/mixed disease who had preoperative left ventricular dysfunction (hazard ratio, 2.3; $P=.03$ ) but not in patients with aortic insufficiency, irrespective of left ventricular function (hazard ratio, $0.7 ; P=.7$ ). In patients with aortic stenosis/mixed disease with left ventricular dysfunction, prosthesis-patient mismatch predicted heart failure symptoms by 3 years after aortic valve replacement (odds ratio, $6.0 ; P=.002$ ), but there was no such effect in patients with aortic insufficiency $(P=.8)$. Indexed left ventricular mass regression occurred to a greater extent in patients with aortic insufficiency than in patients with aortic stenosis/mixed disease (by an additional $29 \pm 5 \mathrm{~g} / \mathrm{m}^{2}, P<.001$ ), and there was a trend for prosthesis-patient mismatch to impair regression in patients with aortic insufficiency (by $\left.30 \pm 17 \mathrm{~g} / \mathrm{m}^{2}, P=.1\right)$.

Conclusions: The incidence and significance of prosthesis-patient mismatch differs in patients with aortic insufficiency compared with those with aortic stenosis or mixed disease. In patients with aortic insufficiency, prosthesis-patient mismatch is seen less frequently and has no significant effect on survival and freedom from heart failure but might have a negative effect on left ventricular mass regression.

Aortic valve replacement (AVR) is the mainstay of treatment for patients with severe aortic valve stenosis, aortic insufficiency (AI), or both. By relieving the obstruction with a competent valve prosthesis, AVR increases survival, improves congestive heart failure (CHF) symptoms, and allows for left ventricular (LV) hypertrophy to regress. ${ }^{1}$ However, the ability of the prosthesis to allow unimpeded blood flow might be suboptimal because of the prosthesis's size, type, and orientation combined with patient factors, such as body size. When present, such a scenario has been called

\footnotetext{
From the Division of Cardiac Surgery ${ }^{\mathrm{a}}$ and the Department of Epidemiology and Community Medicine, ${ }^{\mathrm{b}}$ University of Ottawa Heart Institute, Ottawa, Ontario, Canada.

Received for publication Sept 2, 2008; revisions received Dec 15, 2008; accepted for publication Jan 24, 2009; available ahead of print March 27, 2009.

Address for reprints: Marc Ruel, MD, MPH, Division of Cardiac Surgery, University of Ottawa Heart Institute, 40 Ruskin St, Ottawa, Ontario, Canada K1Y 4W7 (E-mail: mruel@ottawaheart.ca).

J Thorac Cardiovasc Surg 2009;138:639-45

$0022-5223 / \$ 36.00$

Copyright (c) 2009 by The American Association for Thoracic Surgery

doi:10.1016/j.jtcvs.2009.01.013
}

prosthesis-patient mismatch (PPM), and its potential effects continue to be studied in the cardiac surgical literature.

The significance of PPM in patients who have undergone AVR is controversial. Although some have reported that PPM is a rare phenomenon with little effect on patient outcomes, ${ }^{2,3}$ others have shown that PPM is common and associated with decreased LV mass regression, ${ }^{4,5}$ increased recurrence of CHF symptoms, ${ }^{6,7}$ and increased mortality. ${ }^{6,8-12}$ We previously showed that PPM at an indexed effective orifice area (iEOA) equal to or less than $0.85 \mathrm{~cm}^{2} / \mathrm{m}^{2}$ of body surface area (BSA) primarily affects patients with a preoperative left ventricular ejection fraction (LVEF) of less than $50 \%{ }^{10}$; within this group, patients with PPM experienced less complete LV mass regression, less freedom from CHF, and decreased survival.

Indications for AVR include patients with aortic stenosis (AS), AI, or a combination of both (mixed disease). ${ }^{1}$ The heterogeneous nature of the pathology mandating AVR might have been a confounding factor in studies, including our own, that have examined the effect of PPM after AVR. Compared with AS, AI may have distinct causes 


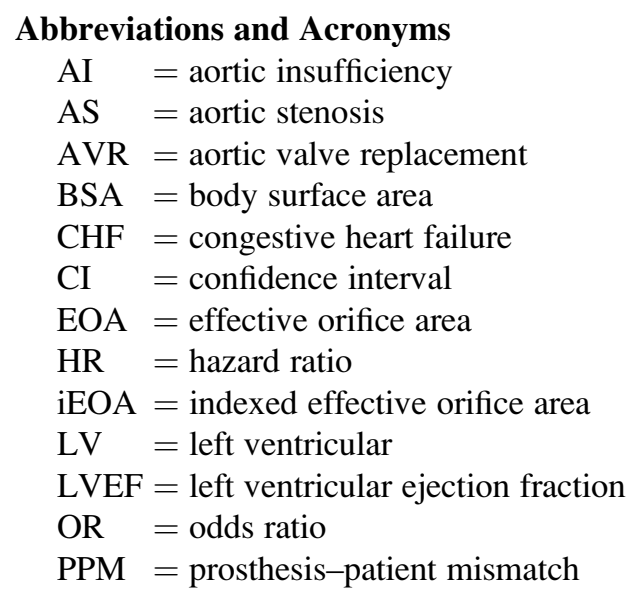

and different effects on the left ventricle; at the time of surgical intervention, the aortic annulus and left ventricle are more often enlarged in patients with AI than in those with AS. Therefore it is conceivable that the incidence of PPM, the extent of post-AVR remodeling, and the clinical and echocardiographic effect of PPM might differ between patients with AI and AS.

To our knowledge, no published study has specifically examined the incidence and effect of PPM in patients with AI versus that seen in patients with AS. We investigated the incidence of PPM and its effects on survival, freedom from CHF and CHF-related death, and LV mass regression in patients with AI compared with those with AS or mixed disease.

\section{MATERIALS AND METHODS \\ Patient Population and Clinical Follow-up}

The patient population consisted of 805 adult patients who underwent AVR at the University of Ottawa Heart Institute between 1990 and 2003. This cohort was the subject of a previous publication. ${ }^{13}$ It has since been updated and further validated through review of individual patient characteristics by 3 external observers. Follow-up was completed to February 1, 2008.

All patients received a prosthesis that is still commercially available in North America. Only patients who survived the operation and were followed up annually at least once in a dedicated valve clinic were included in the analyses. Patients who underwent concomitant mitral valve repair or replacement were excluded. At each clinic visit, patients had a history taken that was focused on the determination of functional status and the occurrence of valve-related complications, a physical examination, electrocardiography, chest radiography, a complete blood count, serum chemistries, and international normalized ratio determinations (when applicable). Patients received anticoagulation treatments according to guidelines in effect at the time, as previously described. ${ }^{14}$

AI was defined as echocardiographic severity of grade 3 or 4 with a mean transvalvular gradient of less than $30 \mathrm{~mm} \mathrm{Hg}$. AS or mixed disease was defined as a patient who did not meet the criteria for pure AI. Persistence or recurrence of heart failure after AVR was defined as the composite end point of New York Heart Association functional class III or IV symptoms for more than 4 consecutive weeks or death for which the primary or contribut- ing diagnosis was $\mathrm{CHF}^{7,9}$ Clinical impressions were corroborated with physical examination, chest radiographic, electrocardiographic, and echocardiographic findings. Prosthesis-related complications were recorded according to the "Guidelines for reporting morbidity and mortality after cardiac valvular operations." 15 The total follow-up was 6174 patient-years, with a mean duration of $7.7 \pm 4.3$ years (range, 60 days- 17.5 years).

\section{Prostheses}

Prosthesis type and size were recorded for all patients. Prostheses were implanted and oriented according to the manufacturer's instructions. The prostheses used were Medtronic Hancock II (Medtronic, Minneapolis, Minn) in 223 (28\%) patients, St Jude Medical Standard (St Jude Medical, St Paul, Minn) in $151(19 \%)$ patients, Carbomedics (Sulzer CarboMedics, Austin, Tex) in 137 (17\%) patients, Medtronic Hall in 89 (11\%) patients, homograft in $58(7 \%)$ patients, Medtronic Hancock I in $47(6 \%)$ patients, St Jude Medical HP in $45(6 \%)$ patients, Edwards Perimount (Edwards Lifesciences, Irvine, Calif) in $38(5 \%)$ patients, MCRI On-X (Medical Carbon Research Institute, Austin, Tex) in $8(1 \%)$ patients, stentless porcine in $5(0.6 \%)$ patients, and Carpentier-Edwards Standard in $4(0.5 \%)$ patients. The in vivo effective orifice area (EOA) for each prosthesis type and size was obtained from the literature of patients with normally functioning prostheses ${ }^{16}$ and averaged if more than 1 published value was available. This was supplemented with data provided by the valve manufacturer if published data were insufficient with respect to a given prosthesis size. The iEOA was obtained by dividing the in vivo EOA by the patient's BSA at the time of the operation and was available for all patients. PPM was defined as an iEOA of $0.85 \mathrm{~cm}^{2} / \mathrm{m}^{2}$ or less for the purpose of examining clinical and echocardiographic outcomes.

\section{Echocardiographic Follow-up}

Patients underwent a complete M-mode, 2-dimensional, Doppler transthoracic echocardiogram before AVR and underwent serial echocardiographic examinations on a biannual basis or as clinically indicated after AVR. Peak instantaneous and mean transvalvular or transprosthesis pressure gradients were derived by using modified Bernoulli equations at each echocardiographic examination. LV end-diastolic and end-systolic diameters, septal and posterior wall thicknesses, and left atrial anteroposterior diameters were measured from the M-mode recordings according to the recommendations of the American Society of Echocardiography. ${ }^{17} \mathrm{LV}$ mass was calculated from these values by using the modified formula of the American Society of Echocardiography. ${ }^{18}$ LVEF was quantified by means of visual estimation by 1 or more of 4 blinded observers. ${ }^{19} \mathrm{LV}$ systolic function was graded as 1 (normal, LVEF $\geq 50 \%$ ), 2 (mildly impaired, LVEF $40 \%$ to $49 \%$ ), 3 (moderately impaired, LVEF $30 \%$ to $39 \%$ ), or 4 (severely impaired, LVEF $<30 \%$ ). Patients were divided into those with normal ( $\mathrm{LVEF} \geq 50 \%$ ) and impaired (LVEF $<50 \%$ ) LV systolic function to assess the effect of PPM according to preoperative LV function.

The latest preoperative echocardiogram and the echocardiogram corresponding to the lowest postoperative LV mass during the follow-up period were used to determine the maximum LV mass regression in each patient. For transprosthesis pressure gradients, we arbitrarily used the echocardiographic examination closest to 18 months postoperatively. This was aimed at allowing LV mass regression to occur while minimizing the likelihood of prosthesis-related issues. ${ }^{10,15}$

\section{Statistical Analyses}

Data were analyzed with Intercooled Stata 10.1 software (StataCorp, College Station, Tex). Continuous data are presented as the mean \pm SD or the mean (lower $95 \%$ confidence limit and upper $95 \%$ confidence limit). Prosthetic valve hemodynamics. Peak and mean instantaneous gradients were compared between patients with and without PPM by using 2-sample $t$ tests within the subgroups of patients with (1) AS or mixed 
disease and normal LV function (LVEF $\geq 50 \%$ ) preoperatively, (2) AS or mixed disease and LV dysfunction (LVEF $<50 \%$ ) preoperatively, (3) AI and normal LV function preoperatively, and (4) AI and LV dysfunction preoperatively.

Survival. Potential predictors of survival, operative indication (including LV function), and PPM were tested for equality with a log-rank test. For multivariate models, the proportional hazard assumption was tested with generalized Cox-Snell residuals. If the assumption was met, Cox proportional hazards models were developed by incorporating variables that had a $P$ value of .05 or less on log-rank testing, by forcing the risk factors for decreased survival after AVR identified previously (ie, age, atrial fibrillation, preoperative heart failure functional class, and coronary artery disease) into the model, ${ }^{7}$ and by incorporating patient characteristics that differed between the mismatch and no-mismatch groups, including sex and the type of prosthesis, into the model. To account for confounding, no automated model selection procedure was used, and all covariates were used simultaneously.

Heart failure. The effects of PPM on the cumulative incidence of heart failure symptoms or heart failure-related death within 3 years after AVR in each of the 4 subgroups of normal and impaired preoperative LV function and valvular lesion, AS, or AI, were examined by using a $\chi^{2}$ test. A 3year period was arbitrarily chosen to allow symptom improvement and $\mathrm{LV}$ remodeling in the greatest potential number of cohort patients ${ }^{7,20}$ while minimizing the possible confounding effects of early structural valve deterioration on heart failure symptoms. ${ }^{21}$ Multivariable logistic regression models incorporated the risk factors for decreased freedom from heart failure after AVR identified previously ${ }^{7}$ and patient characteristics that differed between the groups with and without PPM.

LV mass regression. Echocardiographic LV mass changes were derived from the lowest postoperative echocardiographically derived LV mass minus the preoperative LV mass. These changes were indexed to the patient's preoperative BSA. Changes were compared between patients with and without PPM within the 4 subgroups by using 2-sample $t$ tests.

\section{RESULTS}

Table 1 shows the patient and operative characteristics of the cohort according to the condition that led to AVR. Patients with PPM in the AS or mixed disease group were more often female and older and more often had coronary artery disease. In both the AI and the AS or mixed disease groups, PPM was more often associated with use of a bioprosthetic valve.

\section{PPM Determined by Using In Vivo EOA Values Predicts Prosthetic Valve Hemodynamics After AVR}

At a mean of $1.6 \pm 1.7$ years after surgical intervention, patients with PPM determined on the basis of in vivo EOA (see the Methods section) ${ }^{16}$ had higher peak and mean instantaneous transprosthesis gradients compared with patients without PPM (peak, $29.7 \pm 12.9$ vs $25.2 \pm$ $11.4 \mathrm{~mm} \mathrm{Hg}$; mean, $16.6 \pm 7.3$ vs $13.7 \pm 6.2 \mathrm{~mm} \mathrm{Hg}$, respectively; $P<.001$ ). For patients undergoing operations for AS or mixed disease, the average difference in peak and mean gradients between those with PPM and those without PPM was 3.8 and $2.4 \mathrm{~mm} \mathrm{Hg}$, respectively $(P<.001)$. For patients undergoing operations for AI, the average difference in peak and mean gradients between those with PPM and those without PPM was 10.1 and $6.2 \mathrm{~mm} \mathrm{Hg}$, respectively $(P<.001)$. Within the AS/mixed subgroup and the AI subgroup, PPM had a similar effect on postoperative peak and mean transprosthesis gradients among patients with normal and impaired LV function preoperatively (data not shown).

\section{PPM Is Less Common When AVR Is Performed for AI}

After AVR, the incidence of PPM was significantly lower in patients with $\mathrm{AI}$ (26/98 [26.5\%] patients) than in patients with AS or mixed disease (299/707 [42.3\%] patients, $P=$ $.003)$. A rheumatic cause was present in 3 of the patients with AI, 1 of whom had PPM after AVR.

\section{PPM Negatively Affects Survival, Freedom From \\ Heart Failure, and LV Mass Regression if LV Dysfunction Is Present Before the Operation in Patients With AS but Not in Patients With AI}

Effect on survival. Figure 1 displays the survival of patients with AS or mixed disease after AVR. After adjustment for confounding variables, patients as a whole did not experience significantly worse survival with PPM (hazard ratio $[\mathrm{HR}], 1.4 ; 95 \%$ confidence interval $[\mathrm{CI}], 0.9-2.1 ; P=.1)$. However, if LV dysfunction existed at the time of the operation, PPM was associated with worse survival (HR, 2.3; 95\% CI, 1.1-4.8; $P=.03$ ).

In contrast, this phenomenon was not observed in patients who had AVR for lone AI (Figure 2). In these patients mismatch did not affect survival in the entire group of patients with AI (HR, 0.7; 95\% CI, 0.2-3.4; $P=.7$ ) nor did it affect survival in patients with $\mathrm{AI}$ who had LV dysfunction preoperatively (HR, $1.0 ; 95 \% \mathrm{CI}, 0.1-8.1 ; P=.9$ ).

Effect on freedom from heart failure. The overall incidence of persistent or recurrent CHF symptoms by 3 years after AVR was $11.9 \%$ in patients with AS or mixed disease and $11.5 \%$ in patients with lone $\mathrm{AI}$, respectively $(P=.9)$.

In patients with AS or mixed disease, PPM did not significantly increase the risk of persistent or recurrent CHF symptoms if the LV systolic function was good preoperatively (odds ratio $[\mathrm{OR}]$ for PPM versus no PPM, 1.7; 95\% CI, $0.6-4.6 ; P=.3)$. However, if impaired LV function was present, mismatch was associated with significantly more CHF symptoms (OR, 6.2; 95\% CI, 1.7-22.5; $P=.005)$.

In patients with lone AI, this phenomenon was not observed. Mismatch did not significantly increase the risk of persistent or recurrent $\mathrm{CHF}$ symptoms, regardless of whether the LV systolic function was normal (OR, 2.4; $P=.6)$ or impaired preoperatively $(P=.6)$.

Effect on LV Mass Regression. Figure 3 depicts the extent of maximum $\mathrm{LV}$ mass regression over the follow-up period in the various subgroups. Maximum LV mass regression was observed at a mean of $5.4 \pm 3.5$ years in the AS or mixed disease group and $6.3 \pm 3.8$ years in the lone AI group. 
TABLE 1. Patient characteristics

\begin{tabular}{|c|c|c|c|c|c|c|}
\hline & \multicolumn{2}{|c|}{ Aortic insufficiency } & \multirow[b]{2}{*}{$P$ value } & \multicolumn{2}{|c|}{ Aortic stenosis or mixed disease } & \multirow[b]{2}{*}{$P$ value } \\
\hline & No mismatch $(n=72)$ & $\operatorname{Mismatch}(n=26)$ & & No mismatch $(n=408)$ & $\operatorname{Mismatch}(\mathbf{n}=299)$ & \\
\hline \multicolumn{7}{|l|}{ Preoperative characteristics } \\
\hline Male sex & $56(77.8 \%)$ & $18(69.2 \%)$ & .4 & $292(71.6 \%)$ & $169(56.5 \%)$ & $<.001$ \\
\hline Age $(y)$ & $55.1 \pm 15.1$ & $61.0 \pm 14.7$ & .1 & $59.8 \pm 13.3$ & $71.8 \pm 9.6$ & $<.001$ \\
\hline Body surface area $\left(\mathrm{m}^{2}\right)$ & $1.83 \pm 0.25$ & $1.96 \pm 0.21$ & .01 & $1.82 \pm 0.23$ & $1.94 \pm 0.22$ & $<.001$ \\
\hline Coronary artery disease & $11(15.3 \%)$ & $8(30.8 \%)$ & .1 & $125(30.6 \%)$ & $124(41.5 \%)$ & .003 \\
\hline Atrial fibrillation & $9(12.5 \%)$ & $6(23.1 \%)$ & .2 & $44(10.8 \%)$ & $43(14.4 \%)$ & .2 \\
\hline \multicolumn{7}{|l|}{ NYHA class } \\
\hline I & $14(19.4 \%)$ & $4(15.4 \%)$ & .7 & $103(25.3 \%)$ & $74(24.8 \%)$ & .2 \\
\hline II & $18(25.0 \%)$ & $5(19.2 \%)$ & & $125(30.6 \%)$ & $72(24.1 \%)$ & \\
\hline III & $21(29.2 \%)$ & $11(42.3 \%)$ & & $121(29.7 \%)$ & $109(36.5 \%)$ & \\
\hline IV & $19(26.4 \%)$ & $6(23.1 \%)$ & & $59(14.5 \%)$ & $44(14.7 \%)$ & \\
\hline \multicolumn{7}{|l|}{ Left ventricular grade* } \\
\hline 1 & $49(68.1 \%)$ & $18(69.2 \%)$ & .8 & $266(65.2 \%)$ & $215(71.9 \%)$ & .3 \\
\hline 2 & $10(13.9 \%)$ & $4(15.4 \%)$ & & $72(17.7 \%)$ & $39(13.0 \%)$ & \\
\hline 3 & $10(13.9 \%)$ & $4(15.4 \%)$ & & $41(10.1 \%)$ & $27(9.0 \%)$ & \\
\hline 4 & $3(4.2 \%)$ & 0 & & $29(7.1 \%)$ & $18(6.0 \%)$ & \\
\hline Previous AVR & $25(34.8 \%)$ & $11(42.3 \%)$ & .5 & $66(16.2 \%)$ & $32(10.7 \%)$ & .04 \\
\hline Emergency operation & $1(1.4 \%)$ & $1(4.0 \%)$ & .4 & $11(2.7 \%)$ & $7(2.4 \%)$ & .8 \\
\hline \multicolumn{7}{|l|}{ Operative characteristics } \\
\hline Bioprosthetic valve & $18(25.0 \%)$ & $17(65.4 \%)$ & $<.001$ & $91(22.3 \%)$ & $245(81.9 \%)$ & $<.001$ \\
\hline Aortic root enlargement $\dagger$ & $2(2.8 \%)$ & $2(7.7 \%)$ & .3 & $44(10.8 \%)$ & $30(10.0 \%)$ & .8 \\
\hline
\end{tabular}

Mismatch refers to prosthesis-patient mismatch, which is defined as a ratio of the prosthesis's effective orifice area over the patient's body surface area equal to or less than $0.85 \mathrm{~cm}^{2} / \mathrm{m}^{2}$. $P$ values refer to the comparison between mismatch and no-mismatch patients within the aortic insufficiency and aortic stenosis or mixed disease groups. Data are presented as the mean $\pm \mathrm{SD}$ or number (percentage). NYHA, New York Heart Association; AVR, aortic valve replacement. * Grade 1, Left ventricular ejection fraction of $50 \%$ or more; grade 2, ejection fraction of $40 \%$ to $49 \%$; grade 3, ejection fraction of $30 \%$ to $39 \%$; grade 4 , ejection fraction of less than $30 \%$. $\dagger$ Consists of annular (Nicks, Manougian, or Konno) or aortic root enlargement with pericardium or Dacron.

LV mass regression occurred to a greater degree in patients with AI than in patients with AS or mixed disease (by an additional $29 \mathrm{~g} / \mathrm{m}^{2} ; 95 \% \mathrm{CI}, 18-40 ; P<.001$ ). In patients with AS or mixed disease, there was no significant difference in mass regression as a result of PPM if the left ventricle was normal preoperatively $(P=.9)$. However, in patients with LV dysfunction, PPM was associated with a decrease in the extent of postoperative $\mathrm{LV}$ mass regression (by $\left.20 \mathrm{~g} / \mathrm{m}^{2} ; 95 \% \mathrm{CI}, 6-35 ; P=.007\right)$.

In patients with AI, PPM was associated with a possible decrease in postoperative $\mathrm{LV}$ mass regression (by $29 \mathrm{~g} / \mathrm{m}^{2}$; $95 \% \mathrm{CI},-3$ to $61 ; P=.1$ if the left ventricle was normal; by $33 \mathrm{~g} / \mathrm{m}^{2} ; 95 \% \mathrm{CI},-53$ to $119 ; P=.4$ if the left ventricle was depressed). However, neither scenario reached statistical significance.

\section{DISCUSSION}

The concept of PPM was initially described by Rahimtoola in $1978 .{ }^{22}$ It was defined as the implantation of a prosthesis with an EOA less than that of the normal human aortic valve. Since that time, the definition has evolved to describe a scenario in which the EOA of a prosthesis is too small in relation to patient body size, resulting in abnormally high transprosthesis pressure gradients. ${ }^{23}$ More specifically, most authors have adopted a definition of iEOA of less than 0.85 for PPM. ${ }^{16}$ Others have adopted more stringent definitions $^{8,11}$ or even a continuum of severity with an iEOA of less than 0.65 defined as severe PPM. ${ }^{9,23}$

PPM after AVR has been studied extensively since its description. There is accumulating evidence that this entity is associated with poor outcomes in specific subsets of patients. ${ }^{10,11,13}$ Previously inconsistent results in studies examining the effects of PPM might have been attributable to interaction or confounding from the presence of certain risk factors, such as older age, large body mass index, and pre-existing LV dysfunction. The valve lesion could have been one of these effect-modifying factors as well; in this regard this is the first study to specifically examine the clinical effect of PPM in patients undergoing AVR for AI.

The main findings of this study were that PPM is seen less frequently in patients with AI than in those with AS. In contrast to AS, PPM does not affect clinical outcomes after AVR for AI. However, there might still be suboptimal regression of LV mass in patients with AI and PPM, and this aspect is in need of more study with a greater number of patients.

Specifically, we found that PPM is seen in patients with AI nearly half as frequently as in patients with AS. The overall incidence of PPM was $40 \%$, which is consistent with previous reports. ${ }^{4,9,11,12}$ Although PPM decreased survival in 
Patients Who Had Aortic Valve Replacement for Aortic Stenosis or Mixed Disease

All Patients

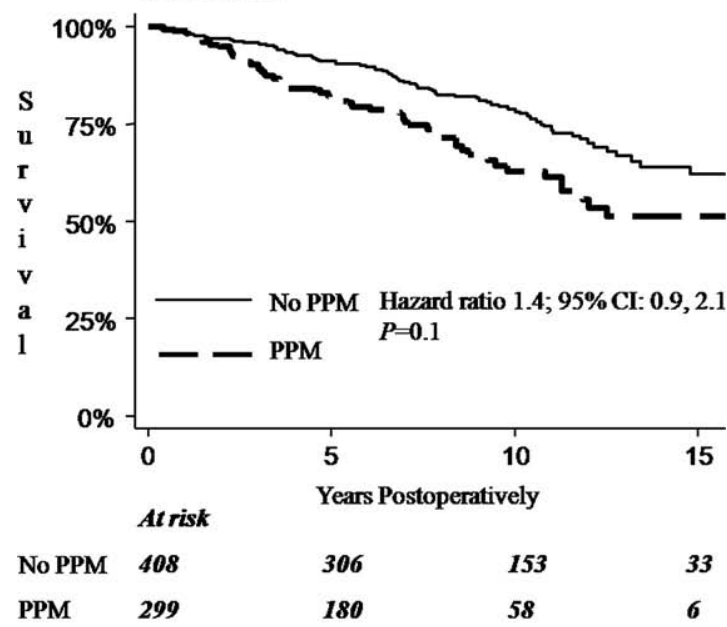

A

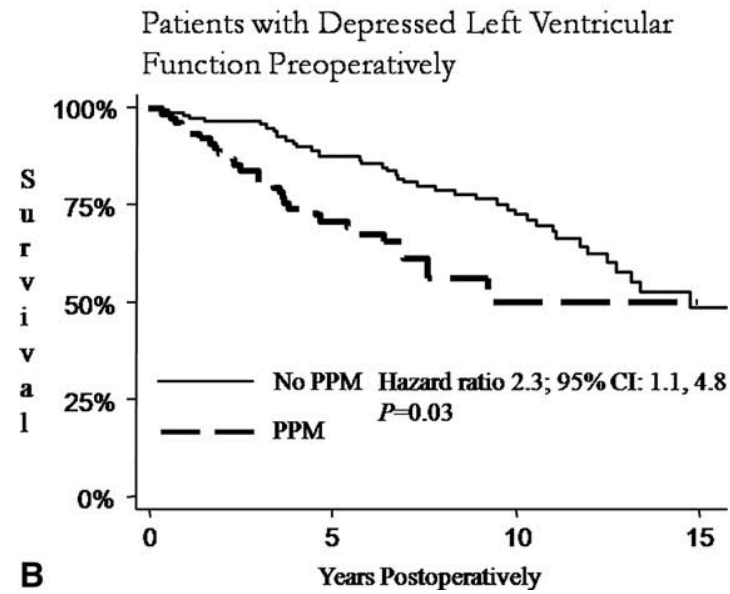

FIGURE 1. Long-term survival after aortic valve replacement with adjusted hazard ratios in all patients with aortic stenosis or mixed disease (A) and patients with aortic stenosis or mixed disease and preoperative left ventricular dysfunction (B). The subset of patients with preoperative left ventricular dysfunction experienced significantly worse long-term survival in the presence of prosthesis-patient mismatch (PPM). 95\% CI, $95 \%$ Confidence interval.

patients with AS with preoperative LV dysfunction, no difference was seen in survival for patients with AI with PPM, regardless of preoperative LV function. Patients with AS with PPM had 6 times the odds of CHF symptoms and death at 3 years if their LVEF was subnormal. This pattern was not seen in patients with AI, regardless of LV function. Only patients with AS with LV dysfunction and PPM had significantly decreased LV mass regression. Interestingly, in patients with $\mathrm{AI}$, there was a trend toward reduced regression in patients with normal LV function and PPM. However, this finding was not statistically significant. Taken together, the results of this study suggest that PPM is encountered
Patients Who Had Aortic Valve Replacement for Pure Aortic Insufficiency

All Patients

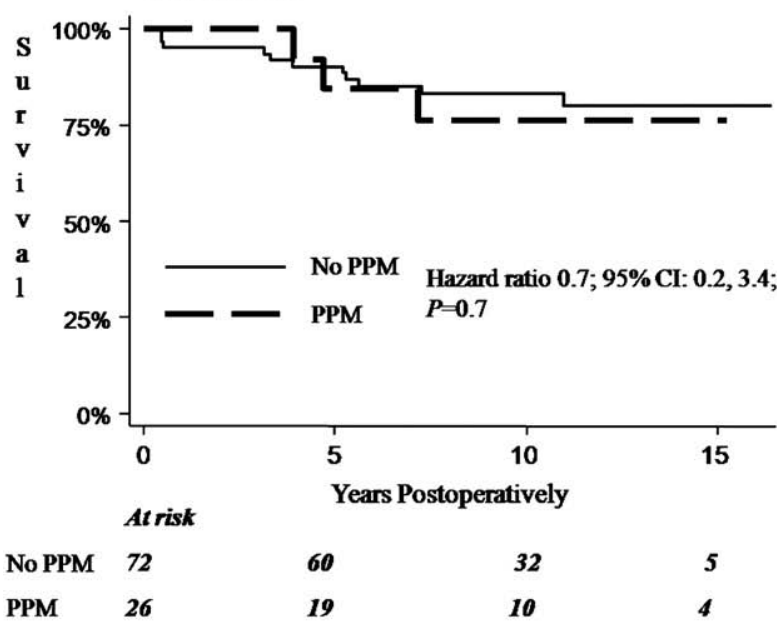

A

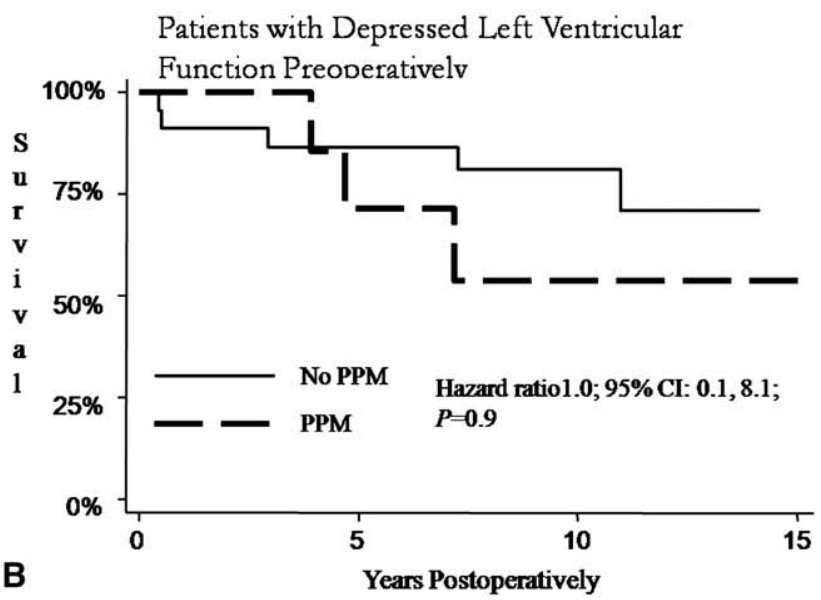

FIGURE 2. Long-term survival after aortic valve replacement with adjusted hazard ratios in all patients with aortic insufficiency (A) and patients with aortic insufficiency with preoperative left ventricular dysfunction (B). Prosthesis-patient mismatch $(P P M)$ did not affect long-term survival in patients with aortic insufficiency. $95 \% C I, 95 \%$ Confidence interval.

less frequently in patients with $\mathrm{AI}$ and is more clinically indolent. Special technical maneuvers to facilitate the implantation of a valve of larger iEOA might increase perioperative morbidity and mortality and therefore do not seem justified in patients with $\mathrm{AI}$ on the basis of improving long-term survival or freedom from CHF symptoms and death.

These data also substantiate our previously published recommendations ${ }^{10}$ that in patients with AS and LV dysfunction, a PPM threshold of 0.85 represents a minimum prosthesis size for optimal survival, freedom from CHF, and LV mass regression after AVR. In patients with AS or mixed disease, measures to ensure implantation of an adequately large prosthesis to avoid PPM seem justified. The effects of PPM on the subgroup of patients with AS and LV 

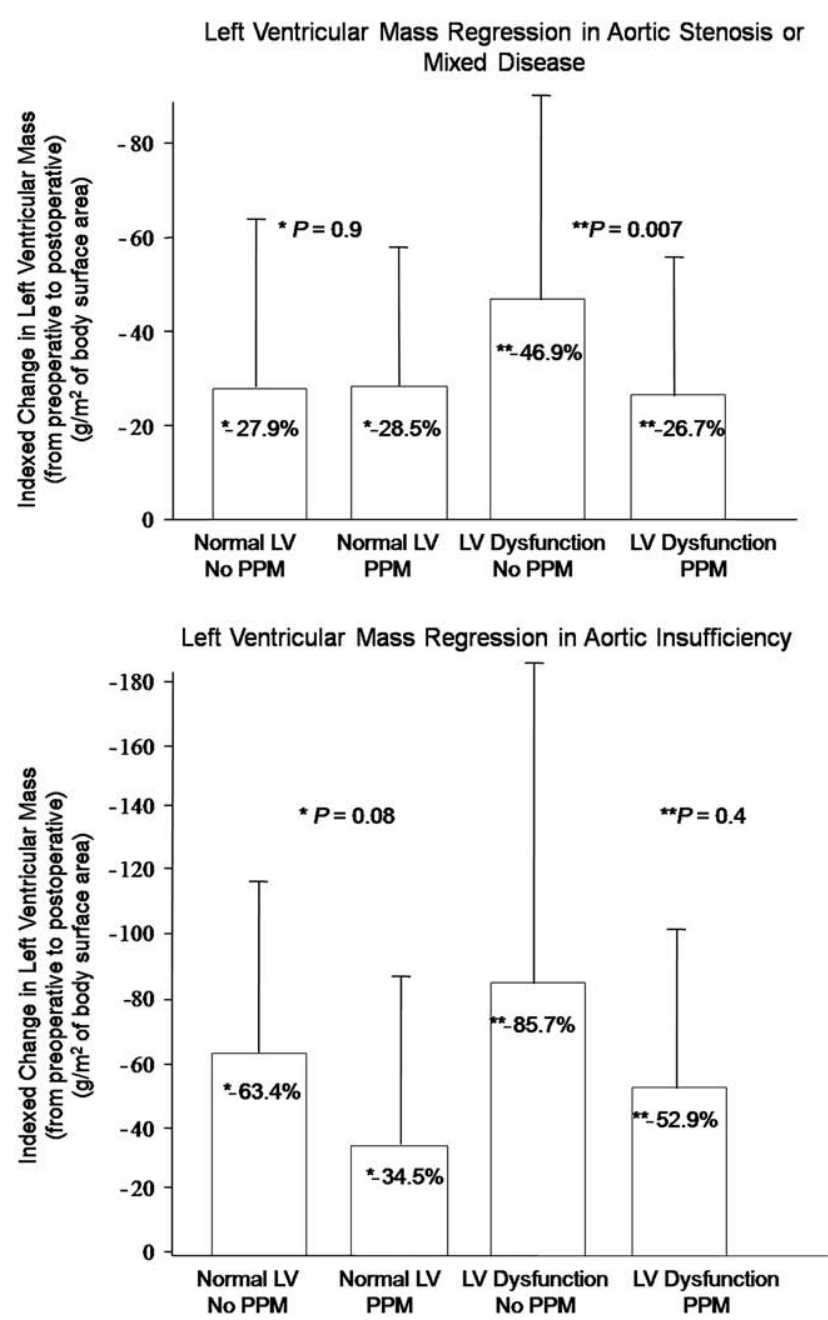

FIGURE 3. Indexed change in left ventricular $(L V)$ mass in patients with aortic stenosis or mixed disease and in patients with aortic insufficiency. In patients with aortic stenosis and left ventricular dysfunction, those with prosthesis-patient mismatch (PPM) experienced a significant reduction in the regression of left ventricular hypertrophy. In patients with aortic insufficiency, a trend toward decreased reduction in left ventricular mass was seen in those with normal left ventricular systolic function and prosthesis-patient mismatch.

dysfunction are consistent with previous findings of our group ${ }^{10}$ and others. ${ }^{9}$ Because the definition of AS included patients with mixed disease and was less stringent than that of AI, the novel and specific findings of this study relate primarily to the outcomes of patients with AI. It is possible that some of the conflicting results observed in previous studies of PPM might be explainable, at least in part, by the effect modification between operative indication and PPM identified in the present data.

\section{Limitations}

In this study we examined the effect of PPM on clinical and echocardiographic outcomes according to LV function by using normal versus decreased LVEF as the dichotomization criterion. We alternatively could have used dichotomization by normal versus enlarged LV end-systolic or end-diastolic dimensions, which are relevant to the management of patients with AI and were used in this study for LV mass determinations. However, although these parameters might be of prognostic importance in patients with $\mathrm{AI}$ as the operative lesion, their use would have limited our ability to make inferences in the subset of patients with AS/mixed disease, who less commonly exhibit LV dilatation. ${ }^{1,24}$

Studies examining PPM are often affected by confounding by indication and selection bias. Surgeons tend to avoid root enlargement techniques or other high-risk maneuvers to implant larger valves in high-risk patients. This might make it spuriously appear that patients receiving smaller valves have worse outcomes. We have attempted to control for these issues through multivariable techniques; however, the relationship between PPM and operative indication observed in this observational study might have been modified by unknown confounders not controlled for in the statistical analyses.

The vast majority of patients in our series had AI based on nonrheumatic causes. These patients tend to have dilated aortic roots, and the results of this study might not be generalizable to patients with rheumatic AI, in whom the aortic annulus tends to be smaller. We believe, however, that these results are valid in the context of operations performed in the Western world, where rheumatic valvular disease is becoming increasingly rare. ${ }^{1}$

We defined and quantified PPM by using the in vivo EOA values derived from normally functioning valves. This method has been used and externally validated in the literature previously ${ }^{25}$; furthermore, it was internally validated in the present study as a strong correlate of actual prosthetic hemodynamics in all subgroups. Using the EOA derived by using the Doppler echocardiographic continuity equation in individual patients after prosthesis implantation has several limitations related to the difficulty of accurately measuring the LV outflow diameter caused by reverberations from the prosthetic valve and the presence of large localized transprosthesis gradients and nonflat transprosthesis spatial velocity profiles, which frequently result in large discrepancies. ${ }^{26-28}$ It was also recently demonstrated that the in vivo EOA method provides superior sensitivity and specificity for prediction of postoperative PPM in individual patients. ${ }^{25}$

\section{CONCLUSIONS}

The incidence of PPM and the effect of PPM on survival, freedom from heart failure, and LV mass regression after AVR are dependent on operative indication. The incidence of PPM in patients with AI after AVR is half of that seen in patients with AS. In contrast to patients with AS, for patients with AI, PPM at a threshold of 0.85 has no effect on survival or freedom from CHF. The results of this study 
support our previous recommendations that in patients receiving AVR for AS, measures to increase the iEOA of the implanted valve should be reserved for the subset of patients with LV dysfunction. In patients with AI, however, technical steps to avoid PPM after AVR do not appear justified solely on the basis of improving postoperative survival or reducing the risk of $\mathrm{CHF}$ or CHF-related death.

\section{References}

1. Bonow RO, Carabello BA, Chatterjee K, de LA Jr, Faxon DP, Freed MD, et al. ACC/AHA 2006 guidelines for the management of patients with valvular heart disease: a report of the American College of Cardiology/American Heart Association Task Force on Practice Guidelines (writing Committee to Revise the 1998 guidelines for the management of patients with valvular heart disease) developed in collaboration with the Society of Cardiovascular Anesthesiologists endorsed by the Society for Cardiovascular Angiography and Interventions and the Society of Thoracic Surgeons. J Am Coll Cardiol. 20061;48:e1-148.

2. Howell NJ, Keogh BE, Barnet V, Bonser RS, Graham TR, Rooney SJ, et al. Patient-prosthesis mismatch does not affect survival following aortic valve replacement. Eur J Cardiothorac Surg. 2006;30:10-4.

3. Flameng W, Meuris B, Herijgers P, Herregods MC. Prosthesis-patient mismatch is not clinically relevant in aortic valve replacement using the Carpentier-Edwards Perimount valve. Ann Thorac Surg. 2006;82:530-6.

4. Fuster RG, Montero Argudo JA, Albarova OG, Sos FH, Lopez SC, Codoner MB, et al. Patient-prosthesis mismatch in aortic valve replacement: really tolerable? Eur J Cardiothorac Surg. 2005;27:441-9.

5. Tasca G, Brunelli F, Cirillo M, DallaTomba M, Mhagna Z, Troise G, et al. Impact of valve prosthesis-patient mismatch on left ventricular mass regression following aortic valve replacement. Ann Thorac Surg. 2005;79:505-10.

6. Mohty-Echahidi D, Malouf JF, Girard SE, Schaff HV, Grill DE, EnriquezSarano ME, et al. Impact of prosthesis-patient mismatch on long-term survival in patients with small St Jude Medical mechanical prostheses in the aortic position. Circulation. 2006;113:420-6.

7. Ruel M, Rubens FD, Masters RG, Pipe AL, Bedard P, Hendry PJ, et al. Late incidence and predictors of persistent or recurrent heart failure in patients with aortic prosthetic valves. J Thorac Cardiovasc Surg. 2004;127:149-59.

8. Rao V, Jamieson WR, Ivanov J, Armstrong S, David TE. Prosthesis-patient mismatch affects survival after aortic valve replacement. Circulation. 2000;102(suppl 3):III5-9.

9. Blais C, Dumesnil JG, Baillot R, Simard S, Doyle D, Pibarot P. Impact of valve prosthesis-patient mismatch on short-term mortality after aortic valve replacement. Circulation. 2003;108:983-8.

10. Ruel M, Al-Faleh H, Kulik A, Chan KL, Mesana TG, Burwash IG. Prosthesis-patient mismatch after aortic valve replacement predominantly affects patients with preexisting left ventricular dysfunction: effect on survival, freedom from heart failure, and left ventricular mass regression. J Thorac Cardiovasc Surg. 2006; 131:1036-44

11. Moon MR, Pasque MK, Munfakh NA, Melby SJ, Lawton JS, Moazami N, et al. Prosthesis-patient mismatch after aortic valve replacement: impact of age and body size on late survival. Ann Thorac Surg. 2006;81:481-8.
12. Walther T, Rastan A, Falk V, Lehmann S, Garbade J, Funkat AK, et al. Patien prosthesis mismatch affects short- and long-term outcomes after aortic valve replacement. Eur J Cardiothorac Surg. 2006;30:15-9.

13. Kulik A, Burwash IG, Kapila V, Mesana TG, Ruel M. Long-term outcomes after valve replacement for low-gradient aortic stenosis: impact of prosthesis-patient mismatch. Circulation. 2006;114(suppl):I553-8.

14. Ruel M, Masters RG, Rubens FD, Bedard PJ, Pipe AL, Goldstein WG, et al. Late incidence and determinants of stroke after aortic and mitral valve replacement. Ann Thorac Surg. 2004;78:77-83.

15. Akins CW, Miller DC, Turina MI, Kouchoukos NT, Blackstone EH, Grunkemeier GL, et al. Guidelines for reporting mortality and morbidity after cardiac valve interventions. J Thorac Cardiovasc Surg. 2008;135:732-8.

16. Pibarot P, Dumesnil JG. Hemodynamic and clinical impact of prosthesis-patient mismatch in the aortic valve position and its prevention. $J$ Am Coll Cardiol. 2000;36:1131-41.

17. Sahn DJ, DeMaria A, Kisslo J, Weyman A. Recommendations regarding quantitation in M-mode echocardiography: results of a survey of echocardiographic measurements. Circulation. 1978;58:1072-83.

18. Devereux RB, Alonso DR, Lutas EM, Gottlieb GJ, Campo E, Sachs I, et al. Echocardiographic assessment of left ventricular hypertrophy: comparison to necropsy findings. Am J Cardiol. 1986;57:450-8.

19. McGowan JH, Cleland JG. Reliability of reporting left ventricular systolic function by echocardiography: a systematic review of 3 methods. Am Heart J. 2003; 146:388-97.

20. Krayenbuehl HP, Hess OM, Monrad ES, Schneider J, Mall G, Turina M. Left ventricular myocardial structure in aortic valve disease before, intermediate, and late after aortic valve replacement. Circulation. 1989;79:744-55.

21. Ruel M, Kulik A, Rubens FD, Bedard P, Masters RG, Pipe AL, et al. Late incidence and determinants of reoperation in patients with prosthetic heart valves. Eur J Cardiothorac Surg. 2004;25:364-70.

22. Rahimtoola SH. The problem of valve prosthesis-patient mismatch. Circulation 1978:58:20-4.

23. Dumesnil JG, Pibarot P. Prosthesis-patient mismatch and clinical outcomes the evidence continues to accumulate. J Thorac Cardiovasc Surg. 2006; 131:952-5.

24. Sasayama S, Ross J Jr, Franklin D, Bloor CM, Bishop S, Dilley RB. Adaptations of the left ventricle to chronic pressure overload. Circ Res. 1976;38 $172-8$.

25. Bleiziffer S, Eichinger WB, Hettich I, Guenzinger R, Ruzicka D, Bauernschmitt R, et al. Prediction of valve prosthesis-patient mismatch prior to aortic valve replacement: which is the best method? Heart. 2007;93:615-20

26. Vandervoort PM, Greenberg NL, Powell KA, Cosgrove DM, Thomas JD Pressure recovery in bileaflet heart valve prostheses. Localized high velocities and gradients in central and side orifices with implications for Doppler-catheter gradient relation in aortic and mitral position. Circulation. 1995;92: 3464-72.

27. Henneke KH, Pongratz G, Bachmann K. Limitations of Doppler echocardiography in the assessment of prosthetic valve hemodynamics. J Heart Valve Dis. 1995;4:18-25.

28. Baumgartner H, Khan S, DeRobertis M, Czer L, Maurer G. Effect of prosthetic aortic valve design on the Doppler-catheter gradient correlation: an in vitro study of normal St. Jude, Medtronic-Hall, Starr-Edwards and Hancock valves. $J$ Am Coll Cardiol. 1992;19:324-32. 